\title{
Disabling Asterixis Induced By Gabapentin
}

\author{
Sarah L. Clark ${ }^{\mathrm{a}}$, Alejandro A. Rabinstein ${ }^{\mathrm{b}}$, Sara E. Hockerb, c
}

\begin{abstract}
We describe a case of an 80-year-old woman who developed disabling asterixis after taking gabapentin for post-herpetic neuropathy. She had end-stage renal disease necessitating intermittent hemodialysis for the previous 5 years. She had begun taking gabapentin about 1 week prior to being admitted to the hospital, and a family member observed a new tremor developing about 3 days prior to hospital admission. Neurologic examination revealed diffuse axial and appendicular negative myoclonus. Because of her renal dysfunction, the gabapentin dose was empirically reduced from $300 \mathrm{mg}$ by mouth three times daily to $100 \mathrm{mg}$ three times daily. Gabapentin was discontinued on hospital day 5 because of concerns for drug toxicity causing the abnormal movements. Two days after gabapentin was discontinued, she has complete resolution of her negative myoclonus.
\end{abstract}

Keywords: Gabapentin toxicity; Asterixis; Adverse drug reaction; Negative myoclonus

\section{Introduction}

Gabapentin is a commonly prescribed medication used for a variety of neurological conditions including migraine headaches, epilepsy, bipolar disorder, anxiety and neuropathic pain [1]. It is generally well tolerated and has limited drug-drug interactions. Gabapentin is exclusively renally eliminated and dosage adjustments are recommended for people with reduced renal function or those receiving hemodialysis [2]. Our report provides a visual illustration of a disabling manifestation of neurotoxicity associated with gabapentin administration in a

Manuscript accepted for publication May 06, 2015

aDepartment of Pharmacy, Mayo Clinic, 200 First Street SW, Rochester, MN 55905, USA

bDepartment of Neurology, Mayo Clinic, 200 First Street SW, Rochester, MN 55905, USA

${ }^{c}$ Corresponding Author: Sara E. Hocker, Department of Neurology, Mayo Clinic, 200 First Street SW, Rochester, MN 55905, USA.

Email: hocker.sara@mayo.edu

doi: http://dx.doi.org/10.14740/jmc2143w hemodialysis-dependent patient.

\section{Case Report}

We present a case of an 80-year-old woman with a new, progressive tremor after initiating gabapentin therapy for a recurrent herpes zoster outbreak. She was transferred to our hospital from an outside facility with a diagnosis of pneumonia after presenting with fevers and dyspnea. Intravenous antibiotics were continued including ceftriaxone and vancomycin. She was hemodynamically stable when she arrived to the hospital. Her pertinent past medical history included hemodialysisdependent end-stage renal disease secondary to hypertensive nephrosclerosis. She was maintained on intermittent hemodialysis three times a week prior to admission for the past 5 years and this was continued throughout her hospital course. Neurology was consulted for evaluation of the "tremor" on hospital day 5. Her examination was remarkable for negative myoclonus of the tongue, extremities and trunk (Supplementary Video, www.journalmc.org). The patient's family reported that the tremor had developed about 3 days prior to her hospitalization but that it had improved since her admission. She had been taking gabapentin $300 \mathrm{mg}$ three times daily for herpetic neuropathy prior to admission. The gabapentin dose was empirically decreased to $100 \mathrm{mg}$ by mouth three times daily on the day of admission to the hospital on the basis of her reduced renal function. Gabapentin was discontinued on hospital day 5 because it was felt to be the only potential cause of her movement disorder. Following discontinuation of gabapentin, the abnormal movements fully resolved within 2 days.

\section{Discussion}

Numerous clinical trials have assessed the efficacy of gabapentin in neuropathic pain and epilepsy. The adverse events associated with gabapentin administration were generally mild to moderate in severity, tended to be self-resolving and dose-related, and infrequently required discontinuation. The most commonly reported neurologic sequelae which occurred in more than $10 \%$ of subjects treated with gabapentin included dizziness, somnolence, fatigue, and tremor, which were also reported in the placebo groups, to a lesser degree. Ataxia was the only 
movement disorder noted in many of the clinical trials, noted in about $7 \%$ of subjects taking gabapentin compared to $0 \%$ in the placebo cohorts $[3,4]$. Other reported neurological toxicities from gabapentin noted in clinical practice include myoclonus, dystonia and chorea, and have been limited to case reports [57]. To date, only three case reports have been published regarding gabapentin-induced asterixis [8-10]. Neurologic toxicity from gabapentin is rare and potentially under-recognized. Literature on serious adverse reactions to gabapentin remains limited to case reports. Because the drug is only excreted by the kidneys, the risk of neurotoxicity from drug accumulation can be higher in patients with renal failure. The normal half-life of gabapentin is between 5 and $7 \mathrm{~h}$, but can be prolonged up to 53 - $152 \mathrm{~h}$ in renal dysfunction or renal failure [2]. Our report provides a visual image of the potential disabling manifestations of neurotoxicity associated with gabapentin use in patients with compromised renal function. The Naranjo adverse drug reaction (ADR) probability score for this case is 7 (probable association) [11]. Serum gabapentin levels were not obtained. Having gabapentin levels would likely have improved the probability score supporting the association.

\section{Financial Support and Disclosure}

None of the authors have pertinent disclosures in regards to this study.

\section{References}

1. Taylor CP. Mechanism of gabapentin. Rev Neurol. 1997;153(suppl 1):539-45.

2. Blum RA, Comstock TJ, Sica DA, Schultz RW, Keller E, Reetze P, Bockbrader H, et al. Pharmacokinetics of gabapentin in subjects with various degrees of renal function. Clin Pharmacol Ther. 1994;56(2):154-159.

3. Rowbotham M, Harden N, Stacey B, Bernstein P, Magnus-Miller L. Gabapentin for the treatment of postherpetic neuralgia: a randomized controlled trial. JAMA. 1998;280(21):1837-1842.

4. Gabapentin in partial epilepsy. UK Gabapentin Study Group. Lancet. 1990;335(8698):1114-1117.

5. Sechi G, Murgia B, Sau G, Peddone L, Tirotto A, Barrocu M, Rosati G. Asterixis and toxic encephalopathy induced by gabapentin. Prog Neuropsychopharmacol Biol Psychiatry. 2004;28(1):195-199.

6. Pina MA, Modrego PJ. Dystonia induced by gabapentin. Ann Pharmacother. 2005;39(2):380-382.

7. Attupurath R, Aziz R, Wollman D, Muralee S, Tampi RR. Chorea associated with gabapentin use in an elderly man. Am J Geriatr Pharmacother. 2009;7(4):220224.

8. Bookwalter T, Gitlin M. Gabapentin-induced neurologic toxicities. Pharmacotherapy. 2005;25(12):1817-1819.

9. Jacob PC, Chand RP, Omeima el S. Asterixis induced by gabapentin. Clin Neuropharmacol. 2000;23(1):53.

10. Babiy M, Stubblefield MD, Herklotz M, Hand M. Asterixis related to gabapentin as a cause of falls. Am J Phys Med Rehabil. 2005;84(2):136-140.

11. Naranjo CA, Busto U, Sellers EM, Sandor P, Ruiz I, Roberts EA, Janecek E, et al. A method for estimating the probability of adverse drug reactions. Clin Pharmacol Ther. 1981;30(2):239-245. 